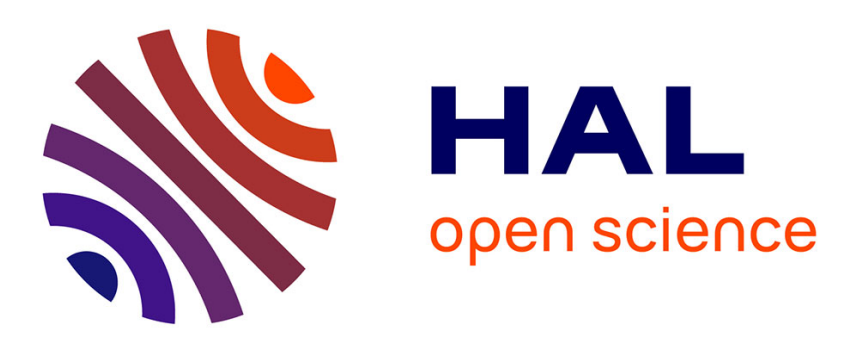

\title{
Toward green three-phase composites with enhanced dielectric permittivity
}

Adel Zyane, François Brouillette, Ahmed Belfkira, Romain Lucas, Pascal Marchet

\section{- To cite this version:}

Adel Zyane, François Brouillette, Ahmed Belfkira, Romain Lucas, Pascal Marchet. Toward green three-phase composites with enhanced dielectric permittivity. Journal of Applied Polymer Science, 2018, 135 (15), pp.46147. 10.1002/app.46147 . hal-01913303

\section{HAL Id: hal-01913303 \\ https://hal-unilim.archives-ouvertes.fr/hal-01913303}

Submitted on 10 Sep 2019

HAL is a multi-disciplinary open access archive for the deposit and dissemination of scientific research documents, whether they are published or not. The documents may come from teaching and research institutions in France or abroad, or from public or private research centers.
L'archive ouverte pluridisciplinaire HAL, est destinée au dépôt et à la diffusion de documents scientifiques de niveau recherche, publiés ou non, émanant des établissements d'enseignement et de recherche français ou étrangers, des laboratoires publics ou privés. 


\title{
Towards green three-phase composites with enhanced dielectric permittivity
}

\author{
Adel Zyane $^{1,2}$, François Brouillette ${ }^{1}$, Ahmed Belfkira ${ }^{2}$, Romain Lucas* ${ }^{3}$, Pascal Marchet ${ }^{3}$ \\ 1 \\ Lignocellulosic Materials Research Centre (CRML), Université du Québec à Trois-Rivières, \\ Box 500, Trois-Rivières, QC, G9A 5H7, Canada \\ 2 \\ Laboratory of Bioorganic and Macromolecular Chemistry (LCBM), Université Cadi Ayyad, \\ Faculté des Sciences et Techniques, Av. Abdelkarim Elkhattabi, B.P 549 Marrakech, Morocco \\ Université de Limoges, SPCTS, UMR 7315, F-87068, Limoges, France
}

Correspondence to: Adel Zyane (E-mail: adel.zyane@uqtr.ca)

\section{ABSTRACT}

This work aims to investigate the dielectric potential of Microcrystalline Cellulose (MCC), a green biosourced material, as a third constituent in the three-phase composites based on Ethylene Vinyl Acetate-Vinyl Ester of Versatic Acid (EVA-VeoVa) Terpolymer and $\mathrm{BaTiO}_{3}$. For that, new green three-phase composites were prepared using an economic and green process, with simple implementation at room temperature and using water as a solvent. Compared with the binary composite EVA-VeoVa/BaTiO ${ }_{3}$, the three-phase composite EVA-VeoVa/BaTiO $/ \mathrm{MCC}$ showed an improvement of the $\mathrm{BaTiO}_{3}$ particles dispersion, enhanced relative permittivity and reduced dielectric loss, which explains the significance of this study.

KEYWORDS: Polymer composites, microcrystalline cellulose (MCC), barium titanate $\left(\mathrm{BaTiO}_{3}\right)$, environmentally friendly composite, dielectric properties. 


\section{INTRODUCTION}

Recently, substantial efforts have been dedicated to find new ways to improve the performance and reduce the weight and volume of dielectric components [1]. In this regard, inorganicorganic systems (such as polymer/ $\mathrm{BaTiO}_{3}$ composites) have received an increasing amount of attention because of their many characteristics, in particular the effect of inorganic phases on polymeric relaxation dynamics [2]. The improvement of the dielectric properties of the polymeric composite depends on the type of fillers added, the nature of the interface between the filler and the polymer, the manufacturing process and the homogeneity of the dispersion of the particles in the matrix $[3,4]$. However, this improvement is observed only when large amounts of inorganic particles are added (generally over to $40 \mathrm{wt} . \%)$. Such high filler levels are detrimental to the mechanical properties of the film [5]. To counter this drawback, the introduction of a third constituent into the polymer/BaTiO 3 system was proposed [6]. Therefore, the third constituent is generally a conductive material (i.e. vapor-grown carbon fibers [7], multi-walled carbon nanotubes [8,9], nanographite [10], graphite nanosheets [11], grapheme [12], $\mathrm{ZnO}$ [13]). The total amount of the third component is much lower than $\mathrm{BaTiO}_{3}$, but it is necessary to achieve a critical concentration of conductive fillers (i.e., the percolation threshold) $[14,15]$. This is required to provide a significant increase in dielectric permittivity while minimizing dielectric losses. According to the literature, the third constituent promotes the interconnection between dipoles, and causes the formation of micro-capacitor networks by separating the neighboring conductive fillers with a thin insulating polymer/ceramic layer $[9,11]$ 
Recent studies have shown that the presence of ethylene vinyl acetate (EVA) as a matrix of composite materials improves the dielectric, barrier and fire retardant properties [16-22]. The success of EVA polymers is due to its ease of fabrication, flexibility, transparency, heat sealability, high adhesivity, processablity and environmentally friendly character $[19,20,23]$. Several studies were conducted to evaluate the dielectric properties of EVA/BaTiO ${ }_{3}$ composites. They report a good interaction between $\mathrm{EVA}$ and $\mathrm{BaTiO}_{3}$ particles, that exhibit a good dispersion and enhanced dielectric properties [24-28]. More recently, environmentally protection has steered research efforts toward natural molecules such as cellulosic fiber and cellulose derivatives. This is due to the advantageous properties of these materials, such as low weight, high strength, free formability and substantial resistance to corrosion and fatigue [29]. Some work focusing on the dielectric properties of hybrid composites based on cellulose was reported, such as rubber/potato starch nanocrystals [30], rubber/nanofibrillated cellulose [31], natural rubber/cellulose II nanocomposites [32]. However, the application of polymer composites with cellulosic fibers in electronics is restrained by their low dielectric properties and the lack of information about morpho-structural aspects and the behavior of such materials when electrical stresses are applied [33].

Microcrystalline cellulose (MCC) is one of the most widely used cellulose derivatives in diverse applications such as medical, pharmaceutical, food, cosmetics and light chemicals [34,35]. MCC is abundant, inexpensive, environmentally friendly and renewable [34]. More importantly, the crystalline structure of this material allows the MCC to perform much better as a reinforcing filler than natural cellulose [36], also this structure has advantages in the formation of a 
parallel-board micro-capacitor network with low filler loading in the ternary composite, in the same manner as conductive fillers [7-13].

In this study, MCC was chosen as the third constituent in the three phase composite based on EVA-VeoVa terpolymer and $\mathrm{BaTiO}_{3}$. Up to now, there has been no report on the preparation of green three-phase composites based on EVA-VeoVa, $\mathrm{BaTiO}_{3}$ and $\mathrm{MCC}$. In addition to the environmentally friendly character of raw materials, we present an economic and green process to produce new dielectric composite materials. The process is simple to implement. It is carried out at room temperature and uses water as a dispersion medium. The addition of MCC should improve the dispersion of $\mathrm{BaTiO}_{3}$ particles and the relative permittivity of the material. The resulting properties of the composite materials will be investigated in comparison with the EVA-VeoVa/BaTiO 3 system.

\section{EXPERIMENTAL}

\section{Materials}

MCC (Avicel PH-105) was received from FMC Europe NV (Brussels, Belgium). The average particle size was $\sim 20 \mu \mathrm{m}$. The latex $\mathrm{W} 301^{\circledR}$ based on EVA-VeoVa terpolymer (referred as VR), was supplied by Société Marocaine des Polymères (SMP). The solid content of the aqueous emulsion was 51,7 wt.\% with a viscosity of $7600 \mathrm{mPa} . \mathrm{s}$ and a glass transition temperature of $34^{\circ} \mathrm{C}$ as indicated by the supplier. Barium titanate $\mathrm{BaTiO}_{3}(\mathrm{BT})$ was synthesized according to our previous work. It has a diameter of 600nm [37]. 


\section{Preparation of the ternary composite}

The base material used to prepare ternary composites consisted in a 80/20 blend of EVA-VeoVa terpolymer (VR) and barium titanate (BT). The preparation technique of the VR/BT binary composite was described previously [37]. The ternary composite was obtained by adding 1 to 4 wt.\% MCC to the VR/BT (80/20) blend. The mixtures were left under mechanical mixing for 30 minutes at room temperature. The obtained dispersions were deposited on Teflon paper under a water-saturated atmosphere. After coalescence and water evaporation, composites were dried for at least $24 \mathrm{~h}$ in a vacuum oven at $70^{\circ} \mathrm{C}$ until a constant weight was reached.

\section{Characterization of the ternary composite}

Scanning Electron Microscope/Energy Dispersive X-Ray Spectrometer (SEM/EDS) images of the composites films were obtained on a JEOL JSM-5500 and OXFORD Instruments, X-Max 20mm², respectively. Gold/Palladium electrodes were deposited on both sides of all samples to ensure a good electrical contact. The dielectric properties of all films were measured in the frequency range from $10^{2} \mathrm{~Hz}$ to $10^{6} \mathrm{~Hz}$ using a precision impedance analyzer (Agilent 4294A). Measurements were taken at different temperatures between $30^{\circ} \mathrm{C}$ and $120^{\circ} \mathrm{C}$.

\section{RESULTS AND DISCUSSION}

\section{Morphology of VR/BT/MCC composite films}

The MCC used in polymer composites has a diameter of $\sim 20 \mu \mathrm{m}$ as shown by the SEM image presented in figure 1a. The chemical structure of the surface of MCC particles promotes interfacial interactions with the VR by creating bridges between hydroxyl groups of MCC and 
EVA-VeoVa polymeric chain dipoles [20,38]. Also, MCC has a much lower specific gravity and a better dispersibility than BT in the initial emulsion and in the dried film. This contributes to the good dispersion of BT in the case of the ternary composite. This effect is shown in Figure 1b-d, which presents SEM images of the binary composite with 20 wt.\% of BT and the ternary composites with 1 and 4 wt.\% of MCC, respectively. The element mapping of different composites (Figure 2) confirms the enhanced dispersion of the BT particles in the matrix obtained by the addition of a small amount of MCC powder. Figure 2a presents some aggregation of BT particles in the VR/BT composite due to the high affinity between filler particles. The addition of $1 \mathrm{wt} . \%$ of MCC contributes to the decrease of these aggregations leading to a more uniform ternary composite as shown in Figure $2 \mathrm{~b}$. The introduction of 4 wt.\% of MCC in the VR/BT composite further improves the dispersion of BT in the matrix (Figure 2c) that confirms the contribution of MCC to the enhancement of BT dispersion. This phenomenon could be explained by the effective interfacial interaction between the three phases, in addition to the hydrophilic character and the high viscosity of the medium (Latex) described in our previous work [37]. In consequence, it is possible to propose the formation of micro-capacitor networks, in which the crystalline areas of MCC act as electrodes and the VR/BT serves as the medium, in the same manner as in the case of PI/graphene/BT [12]. 


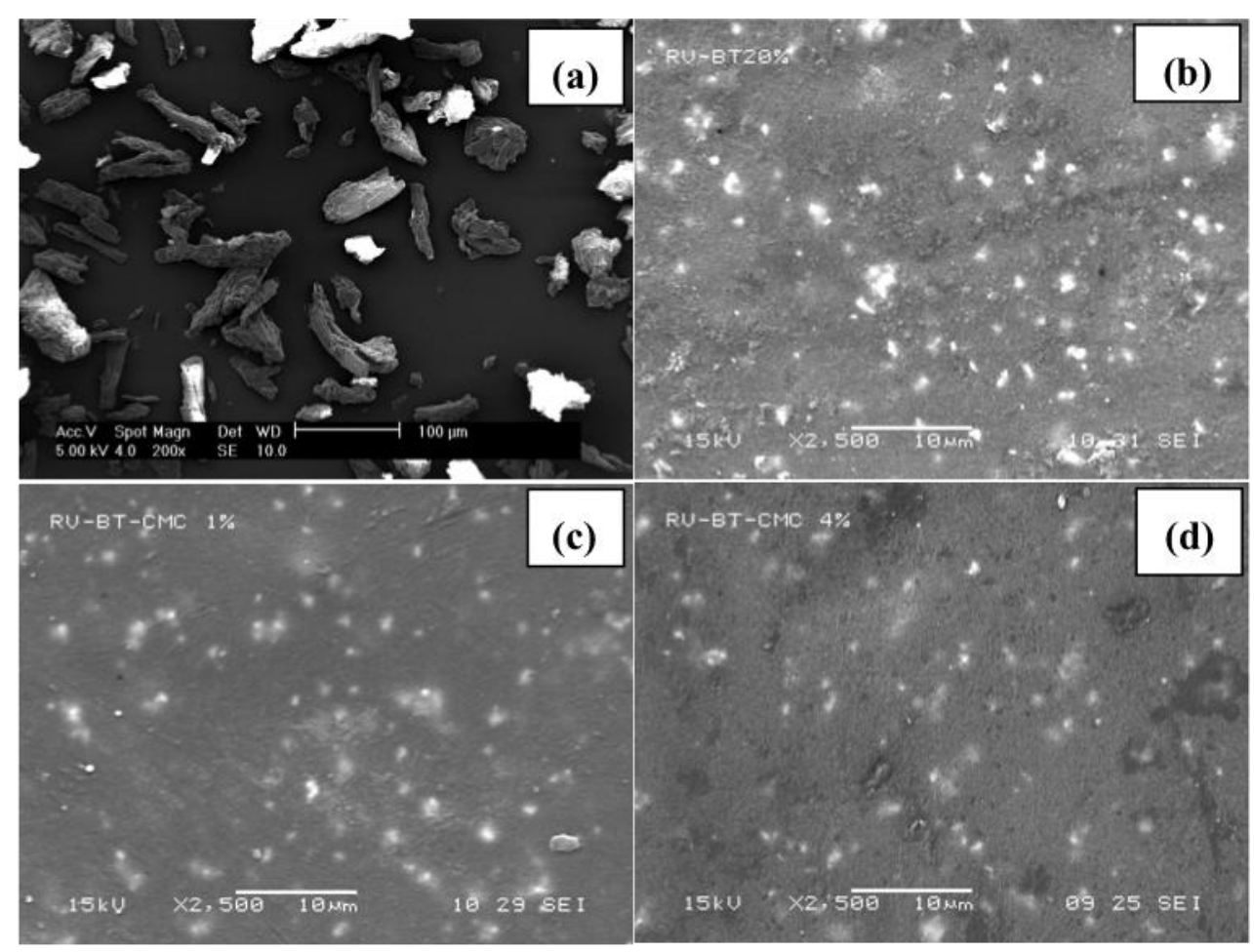

Figure 1: SEM images of (a) MCC powder, (b) VR/BT binary composite with 20 wt.\% of $\mathrm{BaTiO}_{3}$, (c) and (d) VR/BT/MCC ternary composites with 1 and 4 wt.\% of MCC, respectively.

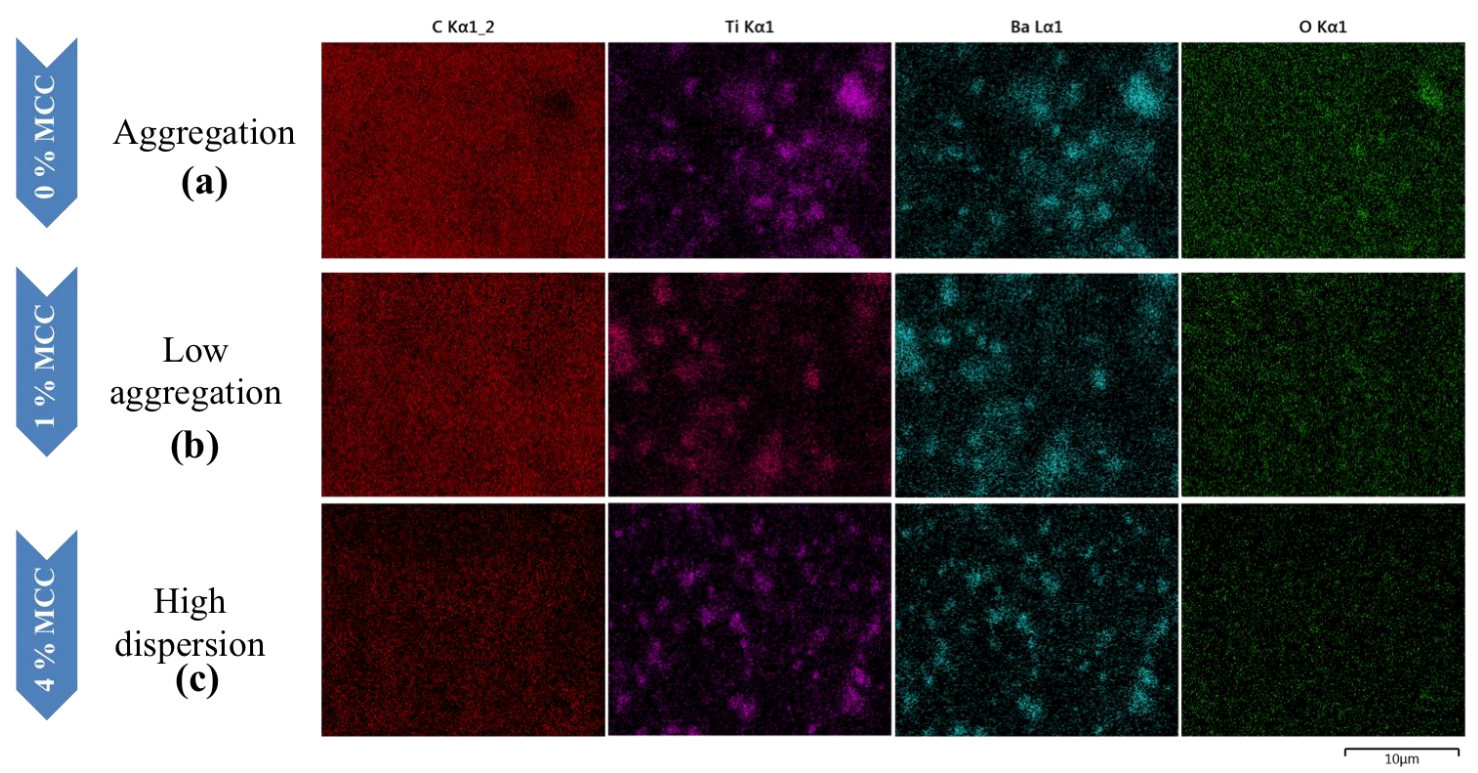

Figure 2: Elements mapping (carbon, titanium, barium and oxygen) of (a) binary composite VR/BT with 20 wt.\% of BT, (b) and (c) ternary composites VR/BT/MCC with 1 wt.\% and 4 wt.\% of $M C C$, respectively. 


\section{Dielectric properties}

Figure 3 presents the temperature dependence of the dielectric permittivity and loss factor of the pure resin and different composites at $1 \mathrm{kHz}$. In the present study, the BT content of the composite films was $20 \mathrm{wt} . \%$, to which an additional amount of 1 to $4 \mathrm{wt} . \%$ MCC was added.

Figure 3a illustrates that the relative permittivity increases as a function of temperature, due to the orientation polarization of the polar groups of the polymer, as explained in our previous work [37]. Also, the dielectric permittivity of the composites increased with MCC concentration for the whole temperature range. VR/BT/MCC composites achieved a dielectric permittivity of 19.15 when the MCC content reached 4 wt.\%, which is 1.8 and 1.5 times higher than that of pure VR and VR/BT 80/20 composite, respectively. These results are also higher than those previously reported for VR/BT composites. In other words, the BT content of the composite can be relatively reduced from 50 to 20 wt.\% by adding only 4 wt.\% of MCC [37].

Dielectric applications require another important parameter, which is the dielectric loss (tanઠ). The temperature dependence of the dielectric loss of the composites, with different amounts of MCC, is shown in Figure 3b. Composites with both BT and MCC show much smaller dielectric losses than the pure VR and the VR/BT composite, which establishes the significance of this study. VR/BT/MCC composites had a tan $\delta$ of 0.012 at low temperature and 0.15 at the maximum of relaxation when the MCC content reached 4 wt.\%. These results are 5.5 and 1.7 times smaller than that of pure VR and VR/BT 80/20 composite, respectively. The reduced dielectric loss is due to the progressive formation of strong bridges between MCC and VR, BT and VR and BT/MCC interfaces. In parallel, the slight leakage of conducive current and the third 
constituent are not connected [12]. Instead of the result reported by Fu-An He et al. for sPS/BaTiO 3 -GNs composites [11], VR/BT/MCC composites do not present conductive paths, which give rise to the leakage of conducive current, responsible for the increase of the dielectric loss. In our case, the incorporation of MCC leads to the formation of successive thin dielectric layers that effectively blocked the leakage current and contributed to the reduction of the dielectric loss. 

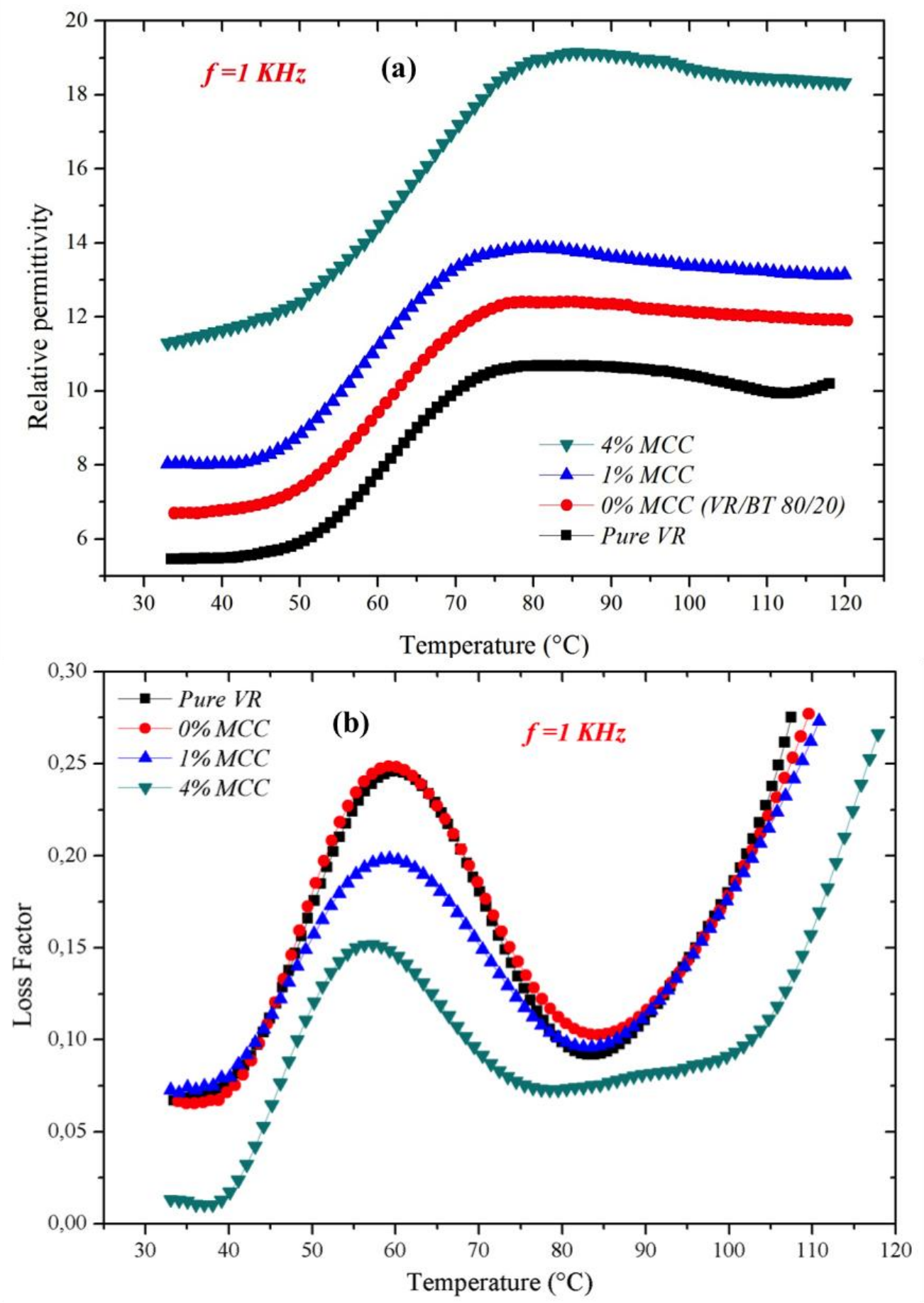

Figure 3: Temperature dependence of (a) dielectric permittivity and (b) loss factor of pure VR and VR/BT/MCC composites with different amounts of MCC at $1 \mathrm{kHz}$. 
Figure 4 represents the dielectric permittivity of the maximum transition (Figure 3) as a function of MCC loading at different frequencies. It can be observed that the dielectric permittivity decreases with increasing frequency. This inverse dependence can be explained experimentally. When the frequency is raised, the dipoles get much less time to orient themselves in the direction of the applied field [37,39]. Also, Figure 4 shows that the dielectric permittivity increases similarly with the increase in MCC loading for all frequencies. This increase can be ascribed to the interfacial polarization (Maxwell-Wagnar effect) originating from the VR/MCC, VR/BT, and BT/MCC interfaces [40]. However, according to the percolation theory, this increase in polarization can be attributed to the existence of several micro-capacitors resulting from the separation of neighboring conductive fillers with a thin insulating polymer/ceramic layer as it was shown for other ternary composites: PVDF/BT/GN and PS/BT/GNs (Graphite Nanosheets) $[10,11]$. In our case, it is possible to imagine the formation of micro-capacitor networks, in which the crystalline zones of MCC act as electrodes and the VR/BT serves as medium. For visual understanding of the dimensional structure of the different constituents in the ternary composite, Figure 5 shows the schematic microstructure evolution processes of VR/BT ( 0 wt.\% of MCC) and VR/BT/MCC ( 1 and 4 wt.\% of MCC). Figure 5a shows that BT particles can be distributed uniformly in the VR matrix with some aggregation in accordance with element mappings (Figure 2). These aggregations decrease when $1 \mathrm{wt} . \%$ of MCC is incorporated in the VR/BT composite, as it can be observed in figure $5 \mathrm{~b}$. The introduction of $4 \mathrm{wt} . \%$ of MCC in the VR/BT composite improves the BT dispersion in the matrix as shown in Figure 5c. However, MCC particles can effectively inhibit the connection of BT particles to increase dispersion of fillers on the one hand and on the others to reduce the leakage current in the BT network and 
endow the film with reduced dielectric loss. Moreover, MCC particles lead to the formation of a micro-capacitor network, which structure is represented with red dash circles in Figure 5c. Therefore, high charges are generated and accumulated in the micro-capacitors when an electric field is applied to the film. This induces the abrupt increase of capacitance and the dielectric permittivity subsequently increases.

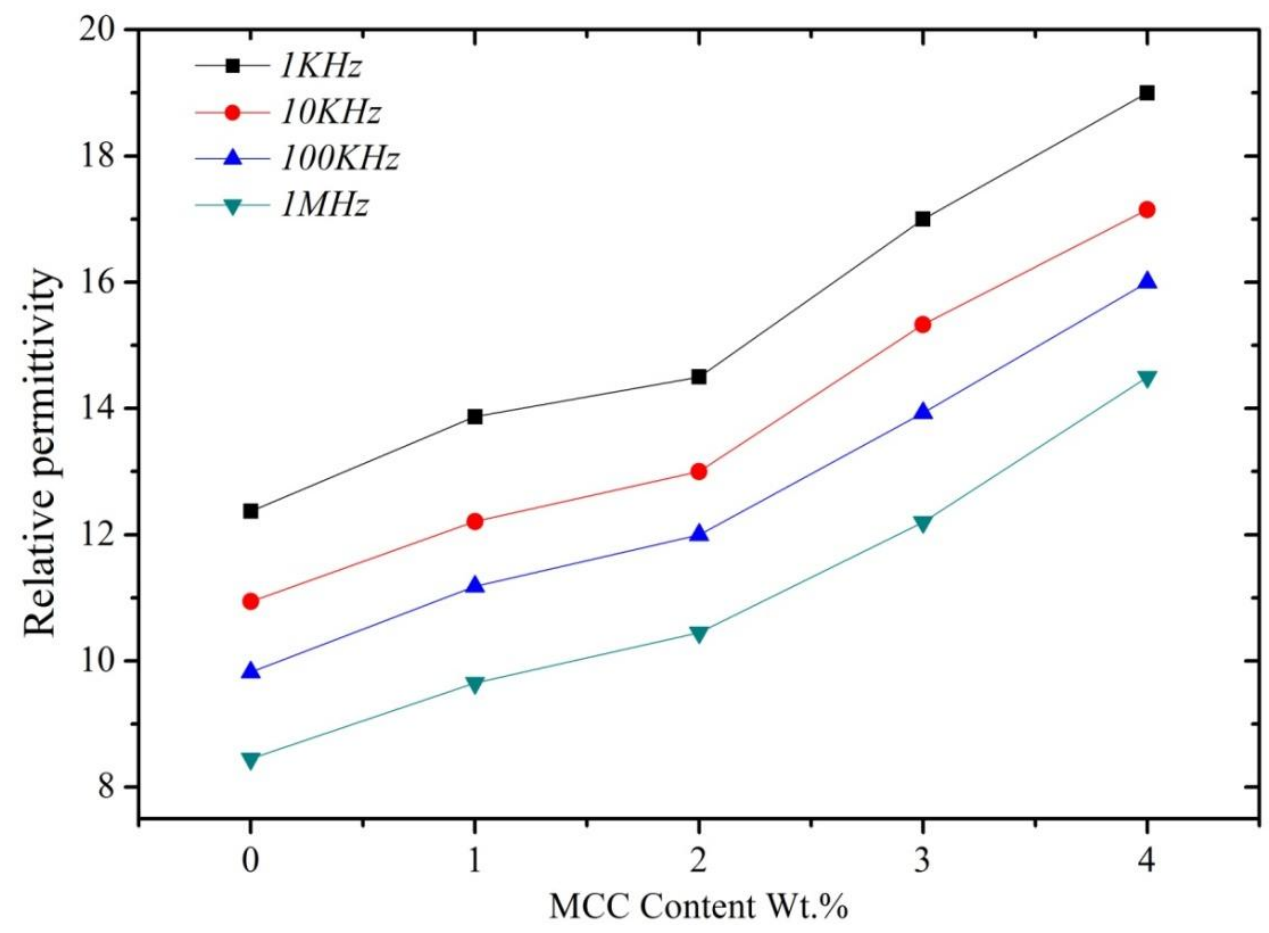

Figure 4: Dielectric permittivity of VR/BT/MCC composites as a function of MCC loading at different frequencies. 

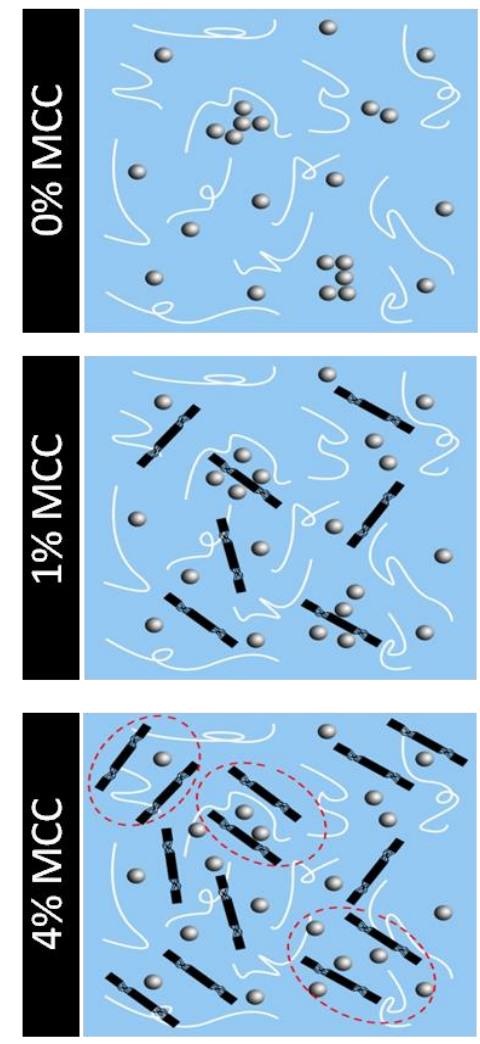

Figure 5: Schematic microstructure of VR/BT ( 0 wt.\% of MCC) and the three-phase composites VR/BT/MCC ( 1 and 4 wt.\% of MCC).

\section{CONCLUSIONS}

Microcrystalline Cellulose (MCC) was chosen as the third constituent in the three-phase composites based on EVA-VeoVa terpolymer (VR) and $\mathrm{BaTiO}_{3}(\mathrm{BT})$ to prepare new green threephase dielectric composites. The addition of MCC to the VR/BT composites leads to a significant enhancement of properties: (i) MCC particles can effectively inhibit the connection of BT particles to increase the dispersion of fillers in the matrix by creating bridges with the composite constituents; (ii) MCC particles promote the formation of a micro-capacitors network that can generate and accumulate high charges when an electric field is applied to the film. This induces an abrupt increase of capacitance and a subsequent increase of the dielectric 
permittivity; (iii) MCC particles lead to the formation of successive thin dielectric layers that

effectively blocked the leakage current and contributed to the reduction of the dielectric loss.

\section{ACKNOWLEDGEMENTS}

This work was financially supported by the "Natural Sciences and Engineering Research Council of Canada (NSERC)" and the "Renewable Materials Research Centre (CRMR)-Canada".

\section{REFERENCES}

1. Dang Z-M, Yuan J-K, Zha J-W, Zhou T, Li S-T, Hu G-H. Fundamentals, processes and applications of high-permittivity polymer-matrix composites. Prog Mater Sci. 2012 May;57(4):660-723.

2. Barber P, Balasubramanian S, Anguchamy Y, Gong S, Wibowo A, Gao H, et al. Polymer Composite and Nanocomposite Dielectric Materials for Pulse Power Energy Storage. Materials. 2009 Oct 29;2(4):1697-733.

3. Wang S-F, Wang Y-R, Cheng K-C, Hsaio Y-P. Characteristics of polyimide/barium titanate composite films. Ceram Int. 2009 Jan;35(1):265-8.

4. Dang Z-M, Xia Y-J, Zha J-W, Yuan J-K, Bai J. Preparation and dielectric properties of surface modified TiO2/silicone rubber nanocomposites. Mater Lett. 2011 Dec;65(23-24):3430-2.

5. Popielarz R, Chiang CK. Polymer composites with the dielectric constant comparable to that of barium titanate ceramics. Mater Sci Eng B. 2007 Apr 25;139(1):48-54.

6. Deng Y, Zhang Y, Xiang Y, Wang G, Xu H. Bi2S3-BaTiO3/PVDF three-phase composites with high dielectric permittivity. J Mater Chem. 2009 Mar 24;19(14):2058-61.

7. Zhang $C$, Sheng JF, Ma CA, Sumita M. Electrical and damping behaviors of CPE/BaTiO3/VGCF composites. Mater Lett. 2005 Dec;59(28):3648-51.

8. Yao S-H, Yuan J-K, Dang Z-M, Bai J. High dielectric performance of three-component nanocomposites induced by a synergetic effect. Mater Lett. 2010 Dec 31;64(24):2682-4.

9. Xu W, Ding Y, Jiang S, Chen L, Liao X, Hou H. Polyimide/BaTiO3/MWCNTs three-phase nanocomposites fabricated by electrospinning with enhanced dielectric properties. Mater Lett. 2014 Nov 15;135:158-61. 
10. Li YC. Dielectric properties of binary polyvinylidene fluoride/barium titanate nanocomposites and their nanographite doped hybrids. Express Polym Lett. $2011 \mathrm{Apr}$ 7;5(6):526-34.

11. He F-A, Lam K-H, Fan J-T, Chan L-W. Novel syndiotactic polystyrene/BaTiO3-graphite nanosheets three-phase composites with high dielectric permittivity. Polym Test. 2013 Aug;32(5):927-31.

12. Liu J, Tian G, Qi S, Wu Z, Wu D. Enhanced dielectric permittivity of a flexible three-phase polyimide-graphene-BaTiO3 composite material. Mater Lett. 2014 Jun 1;124:117-9.

13. Wang G. Enhanced dielectric properties of three-phase-percolative composites based on thermoplastic-ceramic matrix (BaTiO3 + PVDF) and ZnO radial nanostructures. ACS Appl Mater Interfaces. 2010 May;2(5):1290-3.

14. Panda $M$, Srinivas V, Thakur AK. On the question of percolation threshold in polyvinylidene fluoride/nanocrystalline nickel composites. Appl Phys Lett. 2008 Mar 31;92(13):132905.

15. Yao S-H, Dang Z-M, Jiang M-J, Bai J. BaTiO3-carbon nanotube/polyvinylidene fluoride three-phase composites with high dielectric constant and low dielectric loss. Appl Phys Lett. 2008 Nov 3;93(18):182905.

16. Ramesan MT. Dynamic mechanical properties, magnetic and electrical behavior of iron oxide/ethylene vinyl acetate nanocomposites. Polym Compos. 2014 Jan;n/a - n/a.

17. Chaudhary DS, Prasad R, Gupta RK, Bhattacharya SN. Clay intercalation and influence on crystallinity of EVA-based clay nanocomposites. Thermochim Acta. 2005 Aug;433(12):187-95.

18. Riva A, Zanetti M, Braglia M, Camino G, Falqui L. Thermal degradation and rheological behaviour of EVA/montmorillonite nanocomposites. Polym Degrad Stab. 2002 Jan;77(2):299-304.

19. Priya Dasan K, Unnikrishnan G, Purushothaman E. Mechanical, Solvent Imbibing, and Dielectric Behavior of Short Nylon Fiber/Poly(ethylene- co -vinyl acetate) Composites. Adv Polym Technol. 2013 Dec;32(4):n/a - n/a.

20. Sonia A, Priya Dasan K. Celluloses microfibers (CMF)/poly (ethylene-co-vinyl acetate) (EVA) composites for food packaging applications: A study based on barrier and biodegradation behavior. J Food Eng. 2013 Sep;118(1):78-89.

21. Cárdenas MA, García-López D, Gobernado-Mitre I, Merino JC, Pastor JM, Martínez J de D, et al. Mechanical and fire retardant properties of EVA/clay/ATH nanocomposites - Effect of particle size and surface treatment of ATH filler. Polym Degrad Stab. 2008 Nov;93(11):2032-7. 
22. Rahaman M, Chaki TK, Khastgir D. Polyaniline/ethylene vinyl acetate composites as dielectric sensor. Polym Eng Sci. 2013 Jul;n/a - n/a.

23. Zhao F, Li H, Liu S, Chen J. Preparation and properties of an environment friendly polymermodified waterproof mortar. Constr Build Mater. 2011 May;25(5):2635-8.

24. Nagata K, Kodama S, Kawasaki H, Deki S, Mizuhata M. Influence of polarity of polymer on inorganic particle dispersion in dielectric particle/polymer composite systems. J Appl Polym Sci. 1995 Jun 6;56(10):1313-21.

25. Agoudjil B, Ibos L, Candau Y, Majesté J-C. A comparative analysis of dielectric, rheological and thermophysical behaviour of ethylene vinyl acetate/BaTiO ${ }_{3}$ composites. J Phys Appl Phys. 2008 Mar 7;41(5):055407.

26. Huang $X$, Xie L, Jiang P, Wang G, Liu F. Electrical, thermophysical and micromechanical properties of ethylene-vinyl acetate elastomer composites with surface modified $\mathrm{BaTiO}_{3}$ nanoparticles. J Phys Appl Phys. 2009 Dec 21;42(24):245407.

27. Huang $X$, Xie L, Jiang $P$, Shanghai FL. Enhancing the permittivity, thermal conductivity and mechanical strength of elastomer composites by using surface modified BaTiO3 nanoparticles. In IEEE; 2010 [cited 2014 Jun 15]. p. 1-4. Available from: http://ieeexplore.ieee.org/Ipdocs/epic03/wrapper.htm?arnumber=5568234

28. Huang $\mathrm{X}$, Xie L, Hu Z, Jiang P. Influence of $\mathrm{BaTiO}_{3}$ nanoparticles on dielectric, thermophysical and mechanical properties of ethylene-vinyl acetate elastomer/BaTiO microcomposites. IEEE Trans Dielectr Electr Insul. 2011 Apr;18(2):375-83.

29. Jawaid M, Abdul Khalil HPS. Cellulosic/synthetic fibre reinforced polymer hybrid composites: A review. Carbohydr Polym. 2011 Aug 1;86(1):1-18.

30. Bouthegourd E, Rajisha KR, Kalarical N, Saiter JM, Thomas S. Natural rubber latex/potato starch nanocrystal nanocomposites: Correlation morphology/electrical properties. Mater Lett. 2011 Dec;65(23-24):3615-7.

31. Ladhar A, Arous M, Kaddami H, Raihane M, Lahcini M, Kallel A, et al. Dielectric relaxation studies on nanocomposites of rubber with nanofibrillated cellulose. J Non-Cryst Solids. 2013 Oct 15;378:39-44.

32. Ortiz-Serna P, Díaz-Calleja R, Sanchis MJ, Riande E, Nunes R, Martins A, et al. Dielectric spectroscopy of natural rubber-cellulose II nanocomposites. J Non-Cryst Solids. 2011 Jan;357(2):598-604.

33. Panaitescu DM, Notingher PV, Ghiurea M, Ciuprina F, Paven H, lorga M, et al. Properties of composite materials from polyethylene and cellulose microfibrils. J Optoelectron Adv Mater. 2007;9(8):2526-8. 
34. Zuo H-F, Guo Y-R, Li S-J, Pan Q-J. Application of microcrystalline cellulose to fabricate ZnO with enhanced photocatalytic activity. J Alloys Compd. 2014 Dec 25;617:823-7.

35. Gibis M, Schuh V, Weiss J. Effects of carboxymethyl cellulose (CMC) and microcrystalline cellulose (MCC) as fat replacers on the microstructure and sensory characteristics of fried beef patties. Food Hydrocoll. 2015 Mar;45:236-46.

36. Izzati Zulkifli N, Samat N, Anuar H, Zainuddin N. Mechanical properties and failure modes of recycled polypropylene/microcrystalline cellulose composites. Mater Des. 2015 Mar 15;69:114-23.

37. Zyane A, Belfkira A, Brouillette F, Marchet $P$, Lucas R. BaTiO3 incorporation effect on the dielectric properties of polymer from aqueous emulsion: An enhanced dispersion technique. J Appl Polym Sci. 2016 Dec 20;133(48):n/a - n/a.

38. Dongfang Li, Jianzhang Li, Xiaohong Hu, Li Li. Effects of Ethylene Vinyl Acetate Content on Physical and Mechanical Properties of Wood-Plastic Composites. BioResources. 2012 Aug;7(3):2916-32.

39. Sohi NJS, Rahaman M, Khastgir D. Dielectric property and electromagnetic interference shielding effectiveness of ethylene vinyl acetate-based conductive composites: Effect of different type of carbon fillers. Polym Compos. 2011 Jul;32(7):1148-54.

40. Nan C-W, Shen Y, Ma J. Physical Properties of Composites Near Percolation. Annu Rev Mater Res. 2010;40(1):131-51. 
GRAPHICAL ABSTRACT

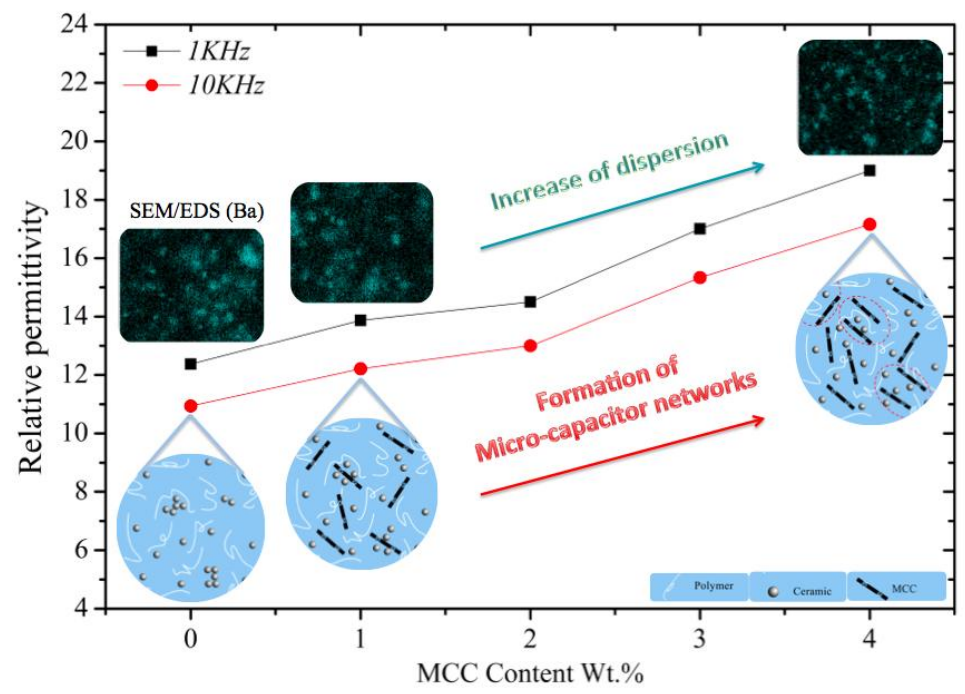

Towards green three-phase composites with enhanced dielectric permittivity

Adel Zyane ${ }^{*}$, François Brouillette, Ahmed Belfkira, Romain Lucas, Pascal Marchet

Microcrystalline Cellulose (MCC), a biosourced material, was chosen as the third constituent to prepare new green three-phase dielectric composites. The addition of MCC improves the dispersion of BT particles and the relative permittivity of composites and contributes to the reduction of the dielectric loss. 